\title{
Being Smart About Gluten and Gluten-Free Issues, Part 1: What Are the Health Concerns Surrounding Gluten?'
}

\author{
Abigail Dicks, Amy Harder, and Amy Simonne ${ }^{2}$
}

Have you noticed food labels in our grocery stores over the last 10 years? They have changed significantly and sometimes read like a science textbook with new nutrition jargon. One newer term we see on products is "gluten free." Are you an inquisitive consumer who asks what the big deal is about gluten free? Ask no more; this article is the first in a three-part series about gluten. The series provides straightforward information for retailers and consumers and basic facts about gluten and gluten free. Part 1 of the series identifies health conditions surrounding the ingestion of gluten. The second publication of the series, What Retailers and Consumers Need to Know About Gluten and Gluten-Free Product Labeling, includes the most current information (at the time of the article) about labeling requirements from the U.S. Food and Drug Administration. Having up-to-date information about gluten-free food labeling regulations will help retailers and consumers alike. The third publication, Marketing Gluten and Gluten-Free Products, addresses specific marketing strategies and activities for retailers. Areas discussed include how to identify the need for gluten-free products in the community, how to select high-quality and safe gluten-free products, how to develop a basic understanding of how gluten-free products are made, how to market the products, and how to build an informed knowledge base with staff and the community.

\section{What makes this information about gluten reliable?}

With a seemingly limitless supply of nutrition information available, few of us have the time or the interest to extract evidence-based nutritional facts from all that is available to us. The resources used in this article are research and evidence based and/or peer reviewed to provide straightforward and simple information about a few gluten-related topics.

\section{Who needs to know versus who wants to know about gluten-free foods?}

Individuals who absolutely need evidence-based information are those with identified food allergies, food intolerances, or food sensitivities, such as gluten. Family members of those with food sensitivities also need to know in order to support the person with the food-related issue. As additional research is conducted, others of us may find ourselves needing a broader understanding of the issues surrounding these topics. We also want to be wise consumers and avoid being swayed by marketing strategies. Before we look specifically at gluten, it is important to understand the differences between a food allergy, a food intolerance, and a food sensitivity (Table 1).

1. This document is WC133, one of a series of the Agricultural Education and Communication Department, Florida Cooperative Extension Service, Institute of Food and Agricultural Sciences, University of Florida. Original publication date January 2013. Visit the EDIS website at http://edis.ifas.ufl. edu.

2. Abigail Dicks, graduate student, and Amy Harder, associate professor, Agricultural Education and Communication Department; Amy Simonne, professor, Department of Family, Youth, and Community Sciences, University of Florida Institute of Food and Agricultural Sciences, Gainesville, FL 32611. 
Table 1. The differences between food sensitivity, food allergy, and food intolerance

\begin{tabular}{|l|l|}
\hline FOOD SENSITIVITY & $\begin{array}{l}\text { Food sensitivity is used as a catch-all term for food allergies and intolerances. Specifically, someone } \\
\text { may be sensitive to histamines or sulfites. Unlikely food sources of histamines may be aged cheeses } \\
\text { and fermented olives. Sulfites may be found in wine and some dried fruits. }\end{array}$ \\
\hline FOOD ALLERGY & $\begin{array}{l}\text { A food allergy is the immune system reacting to a specific protein in foods. It is usually diagnosed } \\
\text { in young children (infants and children under 3 years old). A reaction may be caused by contact } \\
\text { with a tiny amount of the trigger food. Some reactions may be life threatening. A reaction usually } \\
\text { occurs within minutes, or it could be hours later. Most are triggered by one of the eight foods listed } \\
\text { in the bulleted list below. Reactions may change with age and sometimes disappear as a child } \\
\text { gets older. They are sometimes confused with food intolerance. With a food allergy, the immune } \\
\text { system overreacts to a particular food, causing symptoms that are potentially serious or even life } \\
\text { threatening. An allergic reaction is somewhat like a case of mistaken identity by the body's immune } \\
\text { system, which generates antibodies to protect the body against bacteria, viruses, or toxic substances. } \\
\text { There is no cure for food allergies. Strict avoidance of food allergens and early recognition and } \\
\text { management of allergic reactions to food are important measures to prevent serious health } \\
\text { consequences (The Food Allergy \& Anaphylaxis Network, 2011). }\end{array}$ \\
\hline FOOD INTOLERANCE & $\begin{array}{l}\text { Food intolerance is more common than a true food allergy. It does not involve the immune system. } \\
\text { Food intolerance is a metabolism problem in which the body cannot digest the food because of } \\
\text { an enzyme deficiency. Generally, people with food intolerances can eat a small amount of the } \\
\text { food without experiencing major problems. Similar to food allergy symptoms, abdominal cramps, } \\
\text { vomiting, or diarrhea can occur as a result of food intolerance. Unlike food allergies, food intolerance } \\
\text { symptoms may intensify as a person ages. }\end{array}$ \\
\hline
\end{tabular}

\section{Eight foods commonly responsible for food allergies}

- Cow's Milk*

- Eggs

- Fish

- Peanuts

- Shellfish (such as shrimp, prawns, lobster, and crab)

- Tree nuts (such as almonds, cashews, walnuts, pecans, pistachios, Brazil nuts, hazelnuts, and chestnuts)

- Soy*

- Wheat

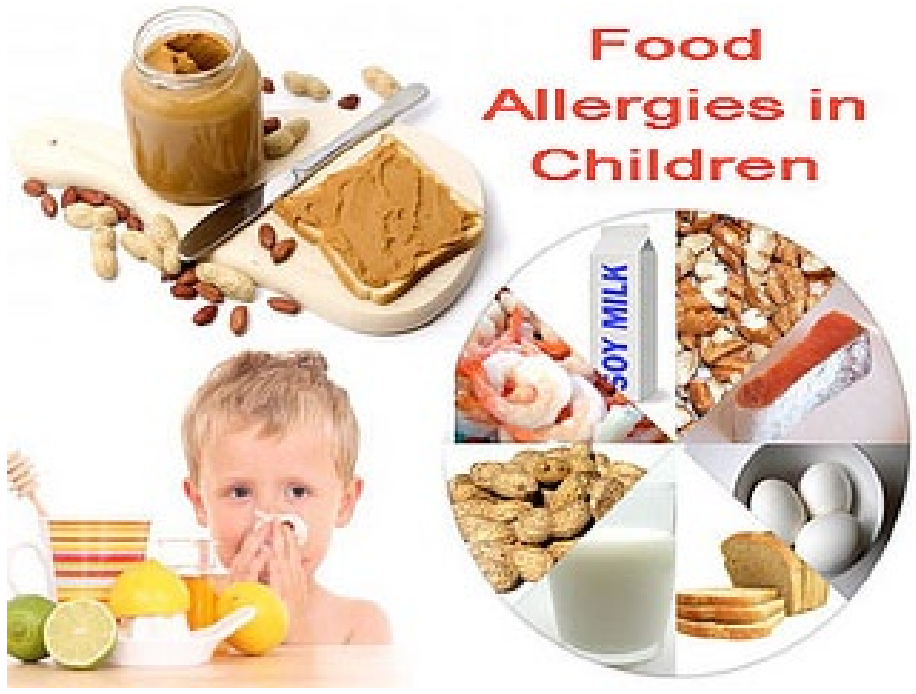

Figure 1. Food allergies

Credits: Adams 999

${ }^{\star}$ Especially among children 


\section{Why do we see more gluten-free food labels now? Why should some individuals care about gluten in their foods?}

Gluten is a mixture of proteins, gliadins, and glutenins found primarily in wheat, rye, or barley. It is known to trigger digestive, immune, or autoimmune systems difficulties for some people (The American Academy of Allergy Asthma and Immunology, 2012). Celiac disease (gluten-sensitive enteropathy) is an autoimmune disease affecting 1 in 130 individuals in the United States (Celiac Sprue Association, n.d.). Celiac disease was once thought to be a rare condition, but with more testing, the incidence has increased significantly, according to the Celiac Sprue Association. It is estimated there are 3,124,000 people in the United States with celiac disease (diagnosed and undiagnosed) and an estimated 70 million people worldwide (The Food Allergy \& Anaphylaxis Network, 2011). Consumer awareness linking gluten (wheat protein) with the onset of celiac disease has prompted consumer interest in knowing which foods contain gluten.

\section{What is celiac disease?}

- Celiac disease is an autoimmune digestive disease that damages the small intestine and interferes with the absorption of important vitamins, minerals, and calories into the body (American Society for Gastrointestinal Endoscopy, 2010).

- Celiac disease is an inherited condition; therefore, family members of a person with celiac disease may wish to be tested (American Society for Gastrointestinal Endoscopy, 2010).

- People with celiac disease cannot tolerate gluten, a protein in wheat, rye, barley, and possibly oats (from cross-contamination) (American Society for Gastrointestinal Endoscopy, 2010).

- A person with celiac disease may or may not have symptoms, which often include diarrhea, abdominal pain and bloating, fatigue, and anemia. Signs and symptoms of the disease classically include diarrhea, short stature, iron-deficiency anemia, and lactose intolerance (American Society for Gastrointestinal Endoscopy, 2010).
- Six to 10 years is the average time a person waits to be correctly diagnosed (National Foundation for Celiac Awareness, 2012).

- Celiac disease is treated by eliminating all gluten from the diet. The gluten-free diet is considered medical nutrition therapy, as well as the only proven treatment that results in improvements in symptomatology and small bowel histology (Pietzak, 2012). The gluten-free diet is a life-long requirement (American Society for Gastrointestinal Endoscopy, 2010).

- Without treatment, people with celiac disease can develop complications like malnutrition, cancer, infertility, osteoporosis, anemia, and seizures (Ansel, n.d.).

- Proper diagnosis includes a small intestinal biopsy and the person's clinical response to a gluten-free diet (American Society for Gastrointestinal Endoscopy, 2010).

- A dietitian working with a gastroenterologist can give detailed guidance and information about food selection, label reading, and other strategies to help manage the disease (American Society for Gastrointestinal Endoscopy, 2010).

- Celiac disease is also known as celiac sprue, nontropical sprue, or gluten-sensitive enteropathy. Celiac disease serves as the best example of delayed hypersensitivity reactions associated with foods (Ansel, n.d.).

\section{What are wheat allergies?}

- If someone has a wheat allergy, the body creates an allergy-causing antibody to a protein found in wheat. In other words, the immune system mistakenly identifies this protein as something that could be harmful. Once the body develops an allergy-causing antibody to a particular agent (allergen) - in this case, a wheat protein - the immune system is sensitive to it. When the person eats wheat, his or her immune system mounts an attack (Mayo Clinic, 2011).

- The branch of the immune system activated in allergic reactions is different from the branch thought to be responsible for the autoimmune reactions of celiac disease. There is usually no severe intestinal damage that may cause nutritional deficiencies (Mayo Clinic, 2011). 
- Allergic reactions after eating wheat may include reactions in the skin, mouth, lungs, and even the gastrointestinal (GI) tract. These can include a rash, wheezing, lip swelling, abdominal pain, and diarrhea.

- Specific activity-related wheat allergies are as follows:

- Wheat-dependent, exercise-induced anaphylaxis occurs when a person with a wheat allergy develops symptoms only if he or she exercises within a few hours after eating wheat. Changes in the body related to exercise either trigger an allergic reaction or worsen an immune system response to a wheat protein. This condition usually results in life-threatening anaphylaxis.

- Baker's asthma is an allergic reaction to wheat flour and other types of flour. It's a particular problem for bakers or anyone who works with uncooked wheat flours. An allergic reaction is triggered by inhaling the flour, resulting primarily in breathing problems.

- A doctor will likely use a combination of tests, including a physical exam, to make a diagnosis. Tests or diagnostic tools may include the following:

- Skin tests - The most common side effects are itching and redness.

- Blood test - This may be done when a skin test can't be performed because of the presence of a skin condition or because of interactions with certain medications. The blood test screens for specific allergy-causing antibodies to various common allergens, including wheat proteins.

- Food diary - This is a detailed record of what and when the person eats, as well as when he or she experiences symptoms.

- Elimination diet - The doctor may recommend a diet with certain foods removed, particularly those foods that are common allergens. Under the doctor's direction, foods are gradually added back, and he or she will note when symptoms return.

- Food challenge testing - This requires the patient to eat capsules containing the food suspected of being the allergy-causing agent. Under careful supervision for a few hours or days, usually at a hospital, the person is given a small amount of the food that is gradually increased. During the test, the patient is monitored for any allergy symptoms

- The best treatment for wheat allergy is to avoid exposure to wheat proteins. Because wheat proteins appear in so many prepared foods, it is necessary to read product labels carefully.

- Antihistamines may reduce signs and symptoms of wheat allergies. These drugs can be taken after exposure to wheat to control the body's reaction and help relieve discomfort.

- Epinephrine is an emergency treatment for anaphylaxis. If a person is at risk for having a severe reaction to wheat, he or she may need to carry two injectable doses of epinephrine.

\section{What is gluten intolerance or gluten sensitivity?}

- In a 2012 study conducted by the Division of Internal Medicine, Hospital of Sciacca, Agrigento, Italy, the existence of nonceliac wheat sensitivity as a clinical entity was established. Nonceliac gluten sensitivity is sometimes confused with celiac disease or thought of as a food allergy. General food intolerances are not thought to be immune system related. There are tests available to identify gluten intolerance or sensitivity (Food Allergy Testing, 2007). Gastrointestinal (GI) symptoms with wheat or gluten intolerance may include gassiness, abdominal pain, abdominal distension, and diarrhea. These symptoms pass once the gluten is out of the person's system and are thought not to cause permanent damage.

- People with allergies and intolerances are thought not to be at an increased risk of developing other autoimmune conditions (Mayo Clinic, 2011).

- People with gluten intolerance usually do not have severe intestinal damage and, therefore, are not at risk for nutritional deficiencies (Mayo Clinic, 2011).

- While avoiding foods containing gluten is a treatment strategy for both food allergy and food intolerance, remember that these are not the same conditions.

- Remember: Wheat free does not necessarily mean gluten free. 

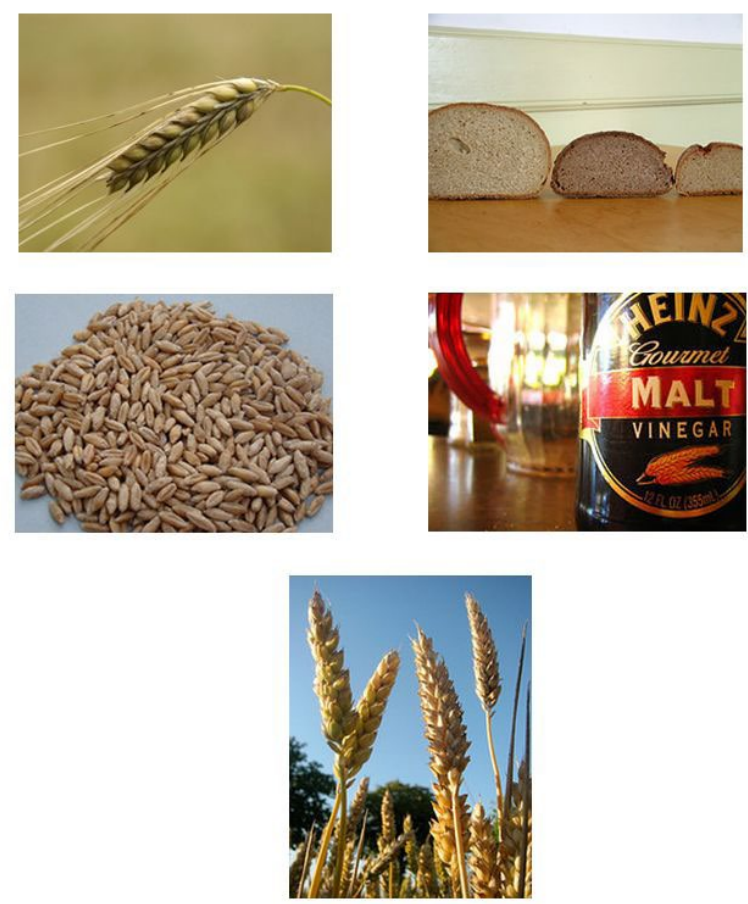

Figure 2. Five main sources of gluten-related health conditions: barley (top left, Credit: freefotouk); rye (top right, Credit: Ib $\tilde{A}_{i} n$ ); triticale (hybrid of wheat and rye) (bottom left, Credit: Agriculturasp); malt, malt flavoring, and malt vinegar (generally made from barley) (bottom right, Credit: gruntusk); and wheat (durum, graham, kamut, semolina, and spelt) (bottom, Credit: Dag Endresen).

\section{My family and I do not have gluten issues, but l'd still like to know...}

Will my family be healthier if we eat gluten free? Limited research has shown nonceliac people did show improvement initially when eating a gluten-free diet, but there are concerns about individuals getting sufficient nutrition on a gluten-free diet and gaining weight from overeating, thinking these foods are okay to eat in quantity.

Why do gluten-free products cost more? We are told that substitutes for wheat flour are harder to get, so it costs more money to produce. It is also a seller's market, which drives prices up. Gluten free started as a specialty niche for people with celiac disease and wheat allergies. Considered a "fad" by some, it has gained in popularity as other health conditions have shown improvement while on a gluten-free diet (Sapone et al., 2012).

\section{Processed foods that may contain hidden gluten as ingredients (Mayo Clinic, 2011)}

- Miso - Miso is fermented soy and wheat mixed together, a common ingredient in miso soup and Asian foods.

- Food products with thickening agents (roux) - Roux is made with fat and flour (wheat flour). It is important for consumers to determine the thickening agents in foods.

- Breading and coating mixes

- Brown rice syrup

- Communion wafers

- Croutons

- Drugs and over-the-counter medications that use gluten as a binding agent

- Energy bars

- Food additives, such as malt flavoring, modified food starch, and others

- Herbal and nutritional supplements

- Imitation bacon or seafood

- Marinades

- Panko (Japanese bread crumbs)

- Play dough - Play dough is a potential problem if hands are put on or in the mouth while playing with it. Hands should be washed immediately after use.

- Processed luncheon meats

- Self-basting poultry

- Stuffing/dressings 
The list below includes other possible hidden sources of gluten (Simonne \& Gollub, 2010):

- Ale/beer/wine/bourbon/whiskey

- Baked goods and mixes (including barley products)

- Battered or breaded foods

- Breakfast cereals

- Candy/chocolate

- Coffee substitutes

- Ice cream and cones

- Pasta/egg noodles

- Pretzels, chips, and crackers

- Soup and soup mixes

- Soy sauce

\section{What is the best buying advice when purchasing gluten-free products?}

Knowing how to read a food label is the key to happily staying gluten free. Here's what you need to know (U.S. Food and Drug Administration, 2011):

- Food labels come in all shapes and sizes. Some are easy to read and provide all the information you need. Others are confusing and leave you with more questions than they answer. When you are trying to follow a gluten-free diet, the most important part of a food label is the ingredients list, usually found on the back or side of the package. In the ingredients list, food companies must accurately list a product's ingredients.

- Be aware of the federal legislation about gluten-free labeling. In 2007, the U.S. Food and Drug Administration proposed the term "gluten free" be used on a voluntary basis to label foods that do not contain any of the following:

- an ingredient that is a species of wheat, rye, barley, or a crossbred hybrid of these grains;

- an ingredient derived from these grains and that has not been processed to remove gluten;

- an ingredient derived from these grains and that has been processed to remove gluten, if the use of that ingredient results in the presence of 20 or more parts per million (ppm) gluten in the food; or
- twenty ppm or more of gluten.

- Very small amounts of ingredients may not be listed on the label; products may also be processed with ingredients (e.g., flour on the conveyor belt) that are not listed on the label or processed in a facility that also processes gluten-containing products that may not always be listed on the label.

- Read labels every time you purchase food. Ingredients can change any time. When in doubt, verify ingredients by calling or writing a food manufacturer and specifying the ingredients and the lot number of the food in question. When calling the manufacturer, state your needs clearly and be patient, persistent, and polite.

\section{Examples of gluten-free foods (Mayo Clinic, 2011)}

- Amaranth

- Arrowroot

- Buckwheat

- Cassava/yucca

- Fresh eggs, meats, fish, and poultry (not breaded, batter-coated, or marinated)

- Fruits and vegetables

- Indian rice grass (Oryzopsis hymenoides)

- Job's tears (Coix lacryma)

- Millet

- Potatoes

- Rice, including wild rice

- Sago

- Protein seeds (quinoa, corn, and flax)

- Soy

- Sorghum

\section{References}

The American Academy of Allergy Asthma and Immunology | AAAAI. (2012). Gluten intolerance defined. Retrieved from http://www.aaaai.org/conditions-and-treatments/ conditions-dictionary/gluten-intolerance.aspx

American Society for Gastrointestinal Endoscopy. (2010, November). ASGE: Celiac disease. Retrieved from http:// www.asge.org/press/press.aspx?id=556 
Ansel, K. (n.d.). Celiac disease. FARRP Home. Retrieved from http://farrp.unl.edu/resources/gi-fas/celiac-disease

Celiac Sprue Association. (n.d.). Retrieved from http:// www.csaceliacs.info/

The Food Allergy \& Anaphylaxis Network. (2011). Food allergy facts and statistics for the U.S. Retrieved from http:// www.foodallergy.org/files/FoodAllergyFactsandStatistics. pdf

Food Allergy Testing. (2007). Food allergy test - food sensitivities test - food intolerance test. Retrieved from http://foodallergytest.org/

Mayo Clinic. (2011). Gluten-free diet: What's allowed, what's not. Retrieved from http://www.mayoclinic.com/ health/gluten-free-diet/MY01140

National Foundation for Celiac Awareness. (2012). Celiac disease facts and figures. Retrieved from http://www. celiaccentral.org/celiac-disease/facts-and-figures/

Pietzak, M. (2012, January). Celiac disease, wheat allergy, and gluten sensitivity: When gluten free is not a fad. Journal of Parenteral and Enteral Nutrition, 36(1 Suppl.), 68S-75S. doi: 10.1177/0148607111426276. Retrieved from http:// www.ncbi.nlm.nih.gov/pubmed/22237879

Sapone, A., Bai, J. C., Ciacci, C., Dolinsek, J., Green, P. H., Hadjivassiliou, M.,...Kaukinen, K. (2012, February). Spectrum of gluten-related disorders: Consensus on new nomenclature and classification. BMC Medicine,10(13). doi: 10.1186/1741-7015-10-13. Retrieved from http://www.ncbi. nlm.nih.gov/pmc/articles/PMC3292448/?tool=pubmed
Simonne, A. H., \& Gollub, E. A. (2010, November). Decoding food labels: Handouts for people with food allergies.

FCS8786. Gainesville: University of Florida Institute of Food and Agricultural Sciences. Retrieved from http://edis. ifas.ufl.edu/pdffiles/FY/FY73000.pdf

U.S. Food and Drug Administration. (2011, August). Questions and answers regarding gluten-free labeling of foods. Retrieved from http://www.fda.gov/Food/LabelingNutrition/FoodLabelingGuidanceRegulatoryInformation/ Topic-SpecificLabelingInformation/ucm265309.htm

\section{Additional Resources}

Carroccio, A., Mansueto, P., Iacono, G., Soresi, M., D’Alcamo, A., Cavataio, F.,...Rini, G. B. (2012). Non-celiac wheat sensitivity diagnosed by double-blind placebocontrolled challenge: Exploring a new clinical entity. American Journal of Gastroenterology, 107(12), 1898-1906. doi: 10.1038/ajg.2012.236. Retrieved from http://www.ncbi. nlm.nih.gov/pubmed/22825366

National Institute of Allergy and Infectious Disease (NIAID). (2010, December). Is it food allergy or food intolerance? Retrieved from http://www.niaid.nih.gov/topics/foodAllergy/understanding/Pages/foodIntolerance.aspx

Norris, J., Barriga, K., Hoffenberg, E., Taki, I., Miao, D., Haas, J.,...Rewers, M. (2005). Risk of celiac disease autoimmunity and timing of gluten introduction in the diet of infants at increased risk of disease. The Journal of the American Medical Association, 293(19), 2343-2351. doi: 10.1001/jama.293.19.2343. Retrieved from http://jama. jamanetwork.com/article. aspx?articleid=200903 